Hautarzt 2014 · 65:8-9

DOI 10.1007/s00105-013-2639-5

Online publiziert: 22. Januar 2014

๑) Springer-Verlag Berlin Heidelberg 2014
G. Daeschlein ${ }^{1} \cdot$ R. Gläser ${ }^{2}$

${ }^{1}$ Hautklinik der Ernst Moritz Arndt Universität, Greifswald

${ }^{2}$ Klinik für Dermatologie, Venerologie und Allergologie,

Universitätsklinikum Schleswig-Holstein, Campus Kiel

\title{
Infektionen in der Dermatologie
}

Trotz zweifellos bedeutender Fortschritte in der Infektiologie und den damit verbundenen tiefer gehenden Kenntnissen über immer mehr Mikroorganismen und deren Auswirkungen im Organismus spielen bakterielle Besiedlungen und Infektionen mit Problemkeimen von Haut und Wunden nach wie vor eine wichtige Rolle und stellen Arzt und Patient immer wieder vor große Herausforderungen. Wund- und Weichteilinfektionen gehören auch heute noch zu den häufigsten Infektionen des Menschen überhaupt, wobei die sich immer mehr verändernde Altersstruktur der Patienten nur einen Teil der Erklärungen liefert, und es wird offensichtlich, dass der medizinische Fortschritt mit diesem globalen Zuwachs an Problemkeimen bisher nicht Schritt halten kann. Sowohl auf der Wirts- als auch auf der Erregerseite sind zwar bereits viele beeinflussende Faktoren bekannt (beim Patienten z. B. Diabetes mellitus, Adipositas und Rauchen), es gibt jedoch auch auf diesem Gebiet noch jede Menge Neues zu erforschen. So stellen z. B. die sich phasenweise abwechselnden Dominanzen bestimmter hochresistenter Keimgruppen bei insgesamt zunehmend gespannter Resistenzlage die Therapie vor immer neue Probleme, ohne dass die Ursachen immer entsprechend nachvollzogen werden können. Erschwerend kommt hinzu, dass aktuell analog zur gegenwärtigen Situation der zunehmenden Verbreitung multiresistenter Gram-negativer Stäbchen [bei relativer Stabilisierung der MRSA (Methicillin-resistente Staphylococcus aureus)-Lage in Europa] diese Problemerreger auch in Wunden vermehrt auftreten. Komplizierend kommt weiter hinzu, dass die ak- tuell im globalen „Aufschwung" befindlichen Gram-negativen Keime just zu dem Zeitpunkt „aktiv“ werden, der sich durch akuten Mangel neuer Antibiotikaentwicklungen auszeichnet. Mit der Empfehlung zur Erfassung der Gram-negativen Stäbchen mit Mehrfachresistenz gegenüber wichtigen Antibiotikaklassen [sog. 3 und 4 MRGN (multiresistente Gram-negative) Stäbchen] hat das Robert Koch-Institut dieser Entwicklung bereits Rechnung getragen und erste Empfehlungen zum Umgang mit diesen Keimen ausgesprochen. Da bisher (u. a. wegen der Besiedlungspositivität im Verdauungstrakt bei „DarmCarriern") keine geeigneten Maßnahmen zur Sanierung empfohlen werden können, gilt das Hauptaugenmerk der Prävention bei Nachweis der Erreger bei Risikopatienten (z. B. Isolierung bei Nachweis von 4 MRGN bei Transplantationspatienten) und der Reduktion der Erregerlast durch Elimination der Keime in Reservoiren, wie z. B. in chronischen Wunden. Zusätzlich sind Anstrengungen vonnöten, durch richtigen Einsatz von Antibiotika weiteren Schaden zu verhüten (Selektion resistenter Erreger) und die Möglichkeiten zu schaffen, um neue Wirkstoffe zu entwickeln.

\section{》) Bakterielle Besiedlungen und Infektionen mit Problemkeimen von Haut und Wunden spielen nach wie vor eine wichtige Rolle}

Ein fundiertes Wissen um die Rolle der verschiedenen Krankheitskeime in den unterschiedlichen klinischen Situationen, ein wirksames, d. h. praxisnahes, ef- fizientes und vermittelbares Hygienemanagement im Rahmen der Infektionsprävention und eine Antibiotikastrategie, die die Selektion resistenter Erreger im Auge behält, sind wichtige Voraussetzungen für den Umgang mit entsprechenden Patienten und ihren Erregern in Praxis und Klinik. Im Rahmen der einzelnen Fachbeiträge zu diesem Themenschwerpunkt rund um Bakterien, Haut und Wunde haben wir gemeinsam mit ausgewiesenen Spezialisten möglichst praxisrelevante Grundlagen und Handlungsanweisungen für Sie zusammengestellt.

Der Artikel von Prof. Dissemond geht klar und übersichtlich auf die klinische Bedeutung, den Nachweis und die Therapie von Bakterien in chronischen Wunden ein.

Einen weiteren Fokus bilden die Staphylokokken und ihre herausragende Rolle als Besiedlungskeime, aber auch hochvirulente Erreger von Haut- und Weichteilinfektionen. Über diese Keime und ihre auch bisher nicht jedem dermatologischen Facharzt allgemein bekannten Eigenschaften [z. B. Bedeutung von "small colony variants" (SCV) bei S. aureus] im Artikel von Prof. Becker et al. zu lesen heißt auch, Infektionen besser zu verstehen und die richtigen Behandlungsund Präventionsmaßnahmen abzuleiten.

Ein weiterer Schwerpunkt wurde in die Wundchirurgie gelegt. Dem Umgang mit postoperativen Wundheilungsstörungen widmet sich die Arbeit von Dr. Nappet al., während die perioperative Prophylaxe als weitere Herausforderung in der täglichen Behandlungspraxis bei Haut- und Weichteilinfektionen von Dr. Dr. Schulze et al. dargestellt wird. 
Umfassend gehen PD Dr. Daeschlein et al. auf verschiedene weitere wichtige Aspekte der mikrobiellen Belastungen von Haut und Wunden in Klinik und Praxis ein. Hier kann jeder Dermatologe weitere praktische Entscheidungshilfen finden, um zwischen „schnellem Handeln“ und „abwartender Haltung“ zu entscheiden.

Ein abschließender interessanter Fokus dieses Leitthemenheftes betrifft die körpereigene Erregerbekämpfung durch antimikrobielle Peptide (AMP). Anschaulich wird von J. Cordes et al. vor Augen geführt, wie bei zahlreichen Situationen mit Infektionsgefährdung durch pathogene Mikroorganismen die antimikrobielle chemische Barriere der Epidermis bereits aktiv wird, bevor die erste gezielte lokale oder systemische antiinfektiöse Therapie eingeleitet wird und greifen kann. AMP eröffnen völlig neue Therapiewege bei Haut- und Wundinfektionen. Eine Strategie könnte hier auch darauf abzielen, die körpereigenen antimikrobiellen Moleküle zu aktivieren und damit die Gabe von Antibiotika und die damit verbundenen unerwünschten Wirkungen zukünftig zu minimieren.

Wir wünschen Ihnen allen viel Freude bei der Lektüre.

PD Dr. Georg Daeschlein

Prof. Dr. Regine Gläser

\section{Korrespondenzadressen}

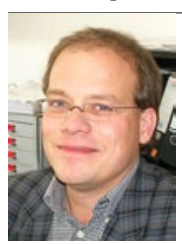

PD Dr. G. Daeschlein

Hautklinik der Ernst Moritz

Arndt Universität

Sauerbruchstraße,

17489 Greifswald

georg.daeschlein@ uni-greifswald.de

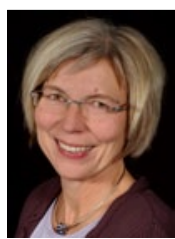

Prof. Dr. R. Gläser

Klinik für Dermatologie, Venerologie und Allergologie, Universitätsklinikum Schleswig-Holstein, Campus Kiel Schittenhelmstr. 7, 24105 Kiel rglaeser@ dermatology.uni-kiel.de

Interessenkonflikt. G. Daeschlein und R. Gläser geben an, dass kein Interessenkonflikt besteht.
Uwe Paasch, Marc Oliver Bodendorf,

Sonja Grunewald

Dermatologische Lasertherapie: Indikationen, Einstellungen, Resultate

Band 1-3

Berlin: KVM 2013, (ISBN 978-3-940698-81-0), Hardcover, 198.00 EUR

Das Leipziger Autorenteam U. Paasch, M. O. Bodendorf und S. Grunewald hat ein dreibändiges Werk "Dermatologische Lasertherapie: Indikationen, Einstellungen, Resultate" im KVM-Verlag Berlin veröffentlicht. Die Federführung des 1. Bandes mit dem Schwerpunkt "Fraktionale Laser" hatte Uwe Paasch, beim 2. Band "Gefäßlaser" Sonja Grunewald und beim 3. Band "Laserepilation" Marc Oliver Bodendorf. Jedes der Bücher folgt dem gleichen klaren Konzept. Für Neulinge auf dem Gebiet werden die Grundlagen des jeweiligen Laserverfahrens inklusive der histologischen Auswirkungen der Therapie konzis dargestellt. Aber auch der erfahrene Anwender findet hier interessante Details. Gerade die Histologie fördert das Verständnis darüber, warum manche Wirkungen auftreten bzw. manche Behandlungen erfolglos bleiben. AnschlieBend wird der konkrete Behandlungsablauf ausführlich beschrieben (Indikationen bzw. Kontraindikationen, Besonderheiten bei der Aufklärung, Geräte- und Parameterwahl usw.). Anhand dieser Informationen kann man den eigenen Behandlungsablauf als „Standard Operating Procedure" reproduzierbar strukturieren. In hervorragenden großformatigen Abbildungen werden danach Ausgangsbefunde, die Laserwirkungen unmittelbar nach der Behandlung sowie im weiteren Verlauf dokumentiert. Dabei werden die einzelnen Indikationen beispielhaft im Bild vorgestellt. Mögliche akute unvermeidbare Behandlungseffekte werden ebenso präsentiert wie unerwünschte akute oder verzögerte Gewebsreaktionen.

Den geübten Anwender erfreut bei der Geräteauswahl eine Übersicht über die physikalischen Parameter nahezu aller gegenwärtig zur entsprechenden Behandlung angebotenen Lasersysteme verschiedener Firmen (z.B. Wellenläge, Energiedichten, Pulslänge, Spotgrößen, Kühlungssysteme und Studien zum jeweiligen Gerät). Eine solche Übersicht zu den angebotenen fraktionalen nicht-ablativen Systemen bzw. fraktionalen ablativen Systemen, zu Gefäß- bzw. Enthaarungsgeräten, muss man sich sonst mühsam zusammensuchen.

Es folgt eine Synopsis der Datenlage zum Erfolg einer Behandlung verschiedener Indikationen mit den genannten Geräten. Die Beurteilung dieser Arbeiten wird durch Angabe eines Evidenzgrads der Studienqualität erleichtert. Die Autoren fassen diese Studien zu jeweils einer Indikation zudem in einem Empfehlungsgrad eines bestimmten Verfahrens als „First-, Second- oder Third-line"-Therapie zusammen, was die Auswahl erheblich erleichtert.

Der erste Band zu den fraktionalen Lasern und der dritte Band zur Laserepilation werden jeweils mit einem Kapitel zu den „Standards in der Lasertherapie" abgeschlossen. Hier werden alle Maßnahmen zusammengefasst, die dabei helfen, Arzt und Patient vor möglichen Fehlern zu schützen und damit reproduzierbar gute Behandlungsergebnisse zu ermöglichen. Dies umfasst auch konkrete Tipps zur guten Fotodokumentation inklusive neuer Verfahren (3-D-Fotografie), zur Lokalanästhesie, zum Kühlen und Absaugen oder zum Fehlermanagement. Auch hier sind alle Empfehlungen konkret und für die Praxis extrem hilfreich. Eine gewisse Redundanz zwischen den Bänden ist dabei wohl unvermeidbar. Im Anhang findet man umfangreiche Literaturverzeichnisse zum Weiterlesen und einen guten Index.

Die Autoren haben drei Bücher vorgelegt, die durch ein klares, einheitliches Konzept, konkrete Hinweise für die tägliche Arbeit, hervorragende Abbildungen und klare Handlungsempfehlungen bestechen. Sie vermitteln einen sehr hohen Praxisnutzen und sind jedem Laseranwender, der entsprechende Indikationen behandelt, uneingeschränkt zu empfehlen.

F. Ochsendorf, Frankfurt 